\title{
BMJ Global Health Effects of a lifestyle intervention programme after 1 year of follow-up among South Asians at high risk of type 2 diabetes: a cluster randomised controlled trial
}

Mirthe Muilwijk (1) , ${ }^{1}$ Marie Loh, ${ }^{2,3}$ Samreen Siddiqui, ${ }^{4}$ Sara Mahmood, ${ }^{5}$ Saranya Palaniswamy, ${ }^{3,6}$ Khurram Shahzad, ${ }^{7}$ Lathika K Athauda, ${ }^{8}$ Ranil Jayawardena, ${ }^{9}$ Tayyaba Batool, ${ }^{10}$ Saira Burney, ${ }^{5}$ Matthew Glover, ${ }^{11}$ Vodathi Bamunuarachchi, ${ }^{12}$ Manju Panda, ${ }^{4}$ Madawa Madawanarachchi, ${ }^{12}$ Baldeesh Rai, ${ }^{13}$ Iqra Sattar, ${ }^{7}$ Wnurinham Silva, ${ }^{13}$ Swati Waghdhare, ${ }^{4}$ Marjo-Riitta Jarvelin, ${ }^{6,13,14,15}$ Ravindra Prasan Rannan-Eliya (D) ,16 Nilmini Wijemunige, ${ }^{16}$ Heather M Gage, ${ }^{17}$ Jonathan Valabhji, ${ }^{18,19}$ Gary S Frost, ${ }^{19}$ Rajitha Wickremasinghe, ${ }^{8}$ Anuradhani Kasturiratne, ${ }^{8}$ Khadija I Khawaja, ${ }^{5}$ Sajjad Ahmad, ${ }^{7}$ Irene GM van Valkengoed, ${ }^{1}$ Prasad Katulanda, ${ }^{20}$ Sujeet $\mathrm{Jha},{ }^{4}$ Jaspal S Kooner, ${ }^{21,22}$ John C Chambers ${ }^{2,3}$

To cite: Muilwijk M, Loh M, Siddiqui S, et al. Effects of a lifestyle intervention programme after 1 year of follow-up among South Asians at high risk of type 2 diabetes: a cluster randomised controlled trial. BMJ Global Health 2021;6:e006479. doi:10.1136/ bmjgh-2021-006479

Handling editor Seye Abimbola

- Additional material is published online only. To view, please visit the journal online (http://dx.doi.org/10.1136/ bmjgh-2021-006479).

Received 1 June 2021 Accepted 30 September 2021

Check for updates

(c) Author(s) (or their employer(s)) 2021. Re-use permitted under CC BY-NC. No commercial re-use. See rights and permissions. Published by BMJ.

For numbered affiliations see end of article.

Correspondence to

Dr Mirthe Muilwijk;

m.muilwijk@amsterdamumc.nl

\section{ABSTRACT}

Introduction South Asians are at high risk of type 2 diabetes (T2D). We assessed whether intensive family-based lifestyle intervention leads to significant weight loss, improved glycaemia and blood pressure in adults at elevated risk for T2D. Methods This cluster randomised controlled trial (iHealthT2D) was conducted at 120 locations across India, Pakistan, Sri Lanka and the UK. We included 3684 South Asian men and women, aged $40-70$ years, without T2D but with raised haemoglobin $\mathrm{A1c}(\mathrm{HbA1c})$ and/or waist circumference. Participants were randomly allocated either to the family-based lifestyle intervention or control group by location clusters. Participants in the intervention received 9 visits and 13 telephone contacts by community health workers over 1-year period, and the control group received usual care. Reductions in weight (aim $>7 \%$ reduction), waist circumference (aim $\geq 5 \mathrm{~cm}$ reduction), blood pressure and $\mathrm{HbA1C}$ at 12 months of followup were assessed. Our linear mixed-effects regression analysis was based on intention-to-treat principle and adjusted for age, sex and baseline values.

Results There were 1846 participants in the control and 1838 in the intervention group. Between baseline and 12 months, mean weight of participants in the intervention group reduced by $1.8 \mathrm{~kg}$ compared with $0.4 \mathrm{~kg}$ in the control group (adjusted mean difference $-1.10 \mathrm{~kg}(95 \% \mathrm{Cl}-1.70$ to -1.06$), \mathrm{p}<0.001)$. The adjusted mean difference for waist circumference was $-1.9 \mathrm{~cm}(95 \% \mathrm{Cl}-2.5$; to 1.3), $\mathrm{p}<0.001)$. No overall difference was observed for blood pressure or HbA1c. People who attended multiple intervention sessions had a dose-dependent effect on waist circumference, blood pressure and $\mathrm{HbA1c}$, but not on weight.

Conclusion An intensive family-based lifestyle intervention adopting low-resource strategies led to effective reduction

\begin{abstract}
WHAT IS ALREADY KNOWN?
$\Rightarrow$ South Asians are at elevated risk for type 2 diabetes (T2D) — diet and physical activity are key modifiable risk factors for T2D and are, therefore, extensively used in lifestyle interventions and guidelines that aim to prevent or delay the onset of T2D.

$\Rightarrow$ Although lifestyle interventions are effective for prevention of T2D in South Asians, the approaches used to date are costly and labour intensive.

\section{WHAT ARE THE NEW FINDINGS?}

$\Rightarrow$ We show that a lifestyle modification delivered by community health workers to South Asians with raised haemoglobin A1c or waist circumference is effective at reducing both weight and waist circumference at 1 year.

$\Rightarrow$ The improvement in weight and waist circumference is generalisable to different cultural groups and geographic settings.

$\Rightarrow$ Our approach is developed to be scalable and sustainable in both low-middle (income) and highincome settings by adopting low-resource-need strategies.
\end{abstract}

in weight and waist circumference at 12 months, which has potential long-term benefits for preventing T2D. A higher number of attended sessions increased the effect on waist circumference, blood pressure and $\mathrm{HbA1C}$.

Trial registration number EudraCT: 2016-001350-18; ClinicalTrials.gov: NCT02949739. 


\section{WHAT DO THE NEW FINDINGS IMPLY?}

$\Rightarrow$ Low-resource strategies such as measuring waist circumference to identify people as being at risk of T2D and the use of community health workers may be used to lower weight and waist circumference among South Asians in both low-middle-income and highincome settings.

$\Rightarrow$ Both weight and waist circumference are on the causal path to T2D; therefore, our approach is likely to reduce the incidence of T2D risk among South Asians.

\section{INTRODUCTION}

Type 2 diabetes (T2D) risk is exceptionally high among South Asians, who represent a quarter of the world population, both in the country of origin and after migration. ${ }^{12}$ Diet and physical activity are key modifiable risk factors for T2D and are, therefore, extensively used in lifestyle interventions and guidelines that aim to prevent or delay the onset of T2D. ${ }^{3-5}$ Recent evidence from a meta-analysis with a total of 1816 participants from six randomised controlled trials (RCTs) (four from Europe and two from India) showed that these types of interventions can be effective to prevent T2D among South Asians as well. ${ }^{6}$

Studies conducted to date have provided some support for the provision of lifestyle intervention for the prevention of T2D among South Asians, however, with significant limitations. First, only few studies were completed in South Asian countries, with no studies reported from Pakistan. Moreover, the completed studies conducted in India ${ }^{78}$ and Sri Lanka ${ }^{910}$ are limited to local settings and small sample sizes. Second, the evidence-based approach established by studies to prevent T2D among South Asians lack scalability and sustainability for T2D prevention, especially in low-middle-income settings. Previous lifestyle interventions to prevent T2D were designed in a way that are labour-intensive and costly, typically including one-to-one sessions with dedicated dieticians and the use of oral glucose tolerance tests for screening. To address these important limitations, we designed the iHealth-T2D trial to improve the scalability and sustainability of lifestyle interventions in both low-middleincome and high-income settings.

The iHealth-T2D trial is a family-based lifestyle intervention encompassing nine visits and 13 telephone calls delivered by community health workers. The iHealth-T2D study aims to (1) identify participants as being at risk for T2D based on parameters, which include low-resource strategies such as measuring waist circumference to identify people at risk and (2) improving cost-effectiveness and scalability through use of community health workers and an intensive family-based lifestyle intervention. Furthermore, we aimed to evaluate the effectiveness of the intervention in different cultural settings in India, Pakistan, Sri Lanka and the UK, to improve generalisability of the research findings. Here, we first evaluate weight, waist circumference, blood pressure and haemoglobin Alc (HbA1c) in the intervention compared with the control arm between baseline and 12 months. In addition, treatment compliance and behavioural changes are evaluated in the intervention arm. Second, we evaluate the effectiveness of low-resource strategies used (raised HbA1c and/or waist circumference) to identify South Asians at risk for T2D subgroup analyses among participants included in the study.

\section{METHODS \\ Study design and participants}

iHealth-T2D is a cluster-RCT among 3684 South Asians at high risk of T2D at 120 locations across India, Pakistan, Sri Lanka and the UK. This is in accordance with power calculations in the study proposal for the primary end point, and these power calculations have been taken into account in the Statistical Analysis Plan (SAP). ${ }^{11}$ The primary end point is new-onset T2D at 3 years. iHealth-T2D was designed to build on previous work, address key limitations in knowledge and explore more scalable approaches to delivery of lifestyle intervention among South Asians. The key innovations making the intervention more scalable compared with previous work, which include identification of South Asians at increased risk of T2D based on waist circumference and HbA1c (HbAlc was recently used to identify participant populations for the first time in the Norfolk Diabetes Prevention Study), ${ }^{12}$ and use of community health workers. A full description of the study design is available in Kasturiratne et al. ${ }^{13}$ In brief, a total of 120 sites from a range of socioeconomic settings and comprising both rural and urban were identified, with 30 sites in each of the four participating countries (India, Pakistan, Sri Lanka and the UK) were included. At each field-work location, we aimed to recruit 15 male and 15 female South Asians aged between 40 and 70 years, at high risk, but free from T2D. Participants were identified at high risk of developing diabetes through screening. The recruitment to the study in India, Sri Lanka and Pakistan comprised inviting individuals living in the community at study sites. Information on the project was distributed, and people were invited for screening through trusted sources of health information including health centres, physicians and healthcare providers, grassroot-level non-physician health workers, accredited social health activists and volunteer groups. People were encouraged to discuss the project with neighbours and friends to help widen engagement. In the UK, participants were exclusively approached by mail, from the practice lists of collaborating general practitioners. A secondary strategy in the UK took advantage of a database comprising the results of cardiovascular health assessments with screening strategies identical to those of the iHealth-T2D study. This approach enabled identification of South Asians meeting the study entry criteria based on HbAlc or waist circumference, who were then invited directly to a study enrolment visit using a single letter of invitation. Screening participants were enrolled into the full trial if they fulfilled the criteria for high risk 
of T2D based on HbA1c $\geq 6.0 \%$ and/or waist circumference $\geq 95 \mathrm{~cm}$ in Sri Lanka, or $\geq 100 \mathrm{~cm}$ in India, Pakistan and the UK. Exclusion criteria were known type 1 or T2D, fasting glucose levels $\geq 7.0 \mathrm{mmol} / \mathrm{L}$, HbAlc levels $\geq 6.5 \%$, body mass index (BMI) $<22 \mathrm{~kg} / \mathrm{m}^{2}$, pregnant or planning pregnancy, unstable residence or planning to relocate and serious illness (eg, cancer). The first participant was enrolled on 15 June 2016 and the last participant on 5 March 2019. The last scheduled 1-year follow-up date was, thus, 4 March 2020.

The primary aim of the lifestyle intervention sessions was the prevention of T2D, through $>7 \%$ wt loss and $\geq 5 \mathrm{~cm}$ reduction in waist circumference. Details on the lifestyle intervention are described in Kasturiratne $e a^{13}$ and are also available on the study website (www.ihealth-t2d.org). In brief, participants in the intervention arm received family-based lifestyle modification (LSM) including all the core Diabetes Prevention Programme design elements. ${ }^{5}$ The lifestyle intervention was delivered by a community health worker and consisted of 22 contact sessions over a period of 12 months: nine clinic visits (four as one-to-one sessions, and five as group sessions), supplemented by 13 telephone contacts. Individuals were counselled on LSM, focusing on improved diet and increased physical activity. Recommendations to improve diet included attention to appropriate portion size, identifying cooking substitutions to reduce fat, increasing fruit and vegetable consumption, decreasing sugar intake and reducing alcohol consumption where appropriate. Suggestions to increase physical activity included finding enjoyable activities to pursue regularly and to incorporate physical activity into daily routines. The target was to achieve $150 \mathrm{~min}$ of moderate physical activity every week. Cultural adaptation was guided by in-country experts in nutrition, dietetics and public health. Comments were sought from potential participants, community health workers and other healthcare providers on draft materials before finalisation. Participants in the control arm received a single diabetes prevention education session lasting 30-60 min delivered by a community health worker, and written material was distributed additionally.

The effectiveness of the iHealth-T2D trial is evaluated after two time points: at 1 and after 3 years of follow-up. Here we report on outcomes after 1 year follow-up. The final outcome (incident T2D) is evaluated after 3 years of follow-up, which will include an evaluation of costeffectiveness. In this current study, we aimed to assess whether the intensive family-based lifestyle intervention programme leads to significant weight loss (weight, waist circumference), improved glycaemia (HbAlc) and blood pressure (systolic and diastolic) in adults at elevated risk for T2D between baseline and 12 months. Treatment compliance and behavioural changes are assessed.

\section{Randomisation and masking}

Neither participants nor community health workers could be blinded to the treatment arm as the type of care given is clearly visible. They were, however, kept masked to outcome measurements and trial results. Data were obtained by research nurses who were blinded to trial arms to reduce the risk of assessment bias. The field-work sites were cluster randomised to either family-based LSM or usual care (1:1 allocation) stratified by country and recruiting centre (two centres in Sri Lanka, five centres in India, two centres in Pakistan). Randomisation was done by the ICL team independent of the local investigators. Participants were enrolled by the study research team and assigned to either the intervention or control arm based on their cluster. The cluster randomisation reduces the risk of resentful demoralisation and Hawthorne effect (contamination) during unblended interventions. ${ }^{14}{ }^{15}$ Cluster randomisation was carried out before recruitment.

\section{Measurements}

Ethnicity was self-reported by the participants. Educational status, based on highest reported education, was used as proxy for socioeconomic status. Weight was measured in light clothing using portable digital scale accurate to $0.1 \mathrm{~kg}$ and height using a portable stadiometer. BMI was determined by dividing measured weight $(\mathrm{kg})$ by height squared $\left(\mathrm{m}^{2}\right)$. Waist circumference was measured over bare skin at the midpoint between the lower margin of the last palpable rib and the top of the iliac crest. Waist measurements were taken three times and the average was calculated. Systolic blood pressure (SBP) and diastolic blood pressure (DBP) $(\mathrm{mm} \mathrm{Hg})$ were measured in the seated position using a digital device (Omron 705, Omron Corporation, Kyoto, Japan). Three measurements were obtained from each participant, 1 min apart, and after a rest period. HbAlc (\%) was determined in fasting (overnight) venous blood samples. HbAlc was measured using Biorad D10 or Variant II turbo assays. Assays were performed in local laboratories that participate in external quality control and meet the standards of the International Federation of Clinical Chemists. Treatment compliance was determined from the number of sessions attended by the participants in the intervention arm. Behavioural changes include assessment of known behavioural variables associated with T2D risk: alcohol consumption, smoking status and physical activity (online supplemental material 1. Alcohol consumption and smoking status were derived from the self-reported questionnaire. Moderate to vigorous-intensity physical activity (metabolic equivalents (MET)/week) and sedentary time ( $\mathrm{min} /$ week) were determined for typical weeks with the Global Physical Activity Questionnaire. ${ }^{16}$

\section{Statistical analyses}

The statistical analyses followed the prespecified SAP. ${ }^{11}$ Statistical analyses were performed by an investigator of the iHealth-T2D study group (MM) who was blinded for the intervention, on a clean anonymised data set (prepared by JC and AK). Analyses were based on the intention-to-treat principle, with data from all participants enrolled in the study, ${ }^{17}$ except for participants who 
withdrew their consent $(n=8)$. Data of participants who attended at least one post-baseline session were analysed according to their initially assigned study arm, regardless of their adherence. Data completeness for baseline variables was almost $100 \%$ and approximately $89 \%$ at 12 months follow-up, mainly due to loss to follow-up. We considered data to be missing at random based on the pattern of missing data. Therefore, multiple imputation was used to impute missing data. Variables selected as predictors for the imputation model contained known predictors of T2D. These include covariates included in the main analysis model (sex, age, country and site) and the auxiliary (baseline) variables (socioeconomic status, smoking status, alcohol consumption, physical activity, waist circumference and HbA1c). Missing values were imputed separately by allocated randomisation group. ${ }^{18}$ The study was well powered to detect changes in study outcomes after 1-year follow-up. Assuming an intraclass correlation coefficient of 0.01 , a two-sided $p$ value of 0.05 , a power of 0.80 and a $0 \%-20 \%$ dropout rate, the detectable differences are $0.80-0.88 \mathrm{~kg}$ in weight, $1.23-1.34 \mathrm{~cm}$ in waist circumference, $1.71-1.87 \mathrm{~mm} \mathrm{Hg}$ in SBP, 0.92$1.00 \mathrm{~mm} \mathrm{Hg}$ in DBP and $0.05 \%-0.06 \% \mathrm{HbAlc}$.

The response rate is determined by dividing the number of people participating in the study by the number of people invited to the study. Baseline characteristics of both study arms are presented in table 1 by sex and country. The baseline characteristics were not tested for statistical differences between study arms. ${ }^{19}$ Baseline characteristics are reported by arithmetic means and SD (normally distributed numerical data), medians and IQR ranges (non-normally distributed numerical data) or by percentages (categorical data). Normality of data distributions was inspected visually by plotting histograms and additionally assessed by the Shapiro-Wilks test.

Differences in continuous outcomes between the two treatment arms were estimated with a multilevel linear mixed-effects regression model, which included the stratification variable site as a random effect and country as fixed effect to adjust for potential cluster differences. Analyses were adjusted for age, sex and baseline values. Including site in the model for weight resulted in high multicollinearity. We, therefore, considered site not to contribute to the model and omitted it in the model for weight. The estimates are presented with their associated 95\% CIs and $p$ values for comparison between the treatment groups. The multiplicative interaction of treatment arm with sex, age, setting, socioeconomic status and baseline waist circumference and $\mathrm{HbA1c}$ levels were assessed. For the outcome weight, the multiplicative interaction with weight instead of waist circumference was assessed, to avoid multicollinearity. In addition, treatment compliance is reported as an explanatory variable in the treatment arm, according to the number of times a participant turned up for the LSM sessions. Behavioural changes were estimated by assessing change from baseline and 12 months in behavioural variables associated with T2D risk (alcohol consumption, smoking and physical activity).
Subgroup analyses of participants included in the study based on a high risk for T2D either according to waist circumference or HbAlc were performed. Sensitivity analyses were performed to identify potentially extreme centres, because extreme deviation of one site from other sites may have a large impact on the overall results. Additionally, complete case analyses were performed to assess the robustness of the results. Finally, any adverse events are reported.

Data are reported in line with the Consolidated Standards of Reporting Trials 2010 Statement. ${ }^{20}$ R V.3.6.3 (R Project for Statistical Computing) was used for the analyses, with the mice package for multiple imputation. ${ }^{21}$ Tests were two-sided and $p$ values $<0.05$ were considered statistically significant. Analyses were not adjusted for multiple testing as hypotheses were pre-set. ${ }^{22}$

\section{Ethics}

Ethical approval was obtained from the Institutional Review Board in each participating country and at each research location before the start of the study. Information sheets and consent forms were made available in the major South Asian languages. Multilingual translators were available as required. Each participant provided informed consent. People unwilling or unable to provide consent were excluded from the study. The research complied with relevant national and international regulations and was carried out in line with the Declaration of Helsinki and the International Conference on Harmonization Guidelines.

\section{Patient and public involvement}

Local communities were involved in the design and adaptation process of the lifestyle intervention. Established trial protocols were tailored for use among South Asians to ensure they were acceptable, suited to the local environment, sustainable and scalable. In South Asia, discussions with community leaders and open meetings were held to identify suitable approaches for engaging local communities in the project. People were encouraged to discuss the project with neighbours and friends to help widen engagement. Family members of participants were strongly encouraged to join the programme, in particular, the children and the family $\operatorname{cook}(\mathrm{s})$. Family members were encouraged to make healthier choices, but without specific targets for weight and/or waist circumference unless they were also obese. Results of the study will be shared with study participants through the study website, and flyers and brochures will be circulated. Local media will be engaged to release press reports. Moreover, we will actively participate in local events focused on T2D and healthy lifestyle.

\section{Role of the funding source}

The funder of the study had no role in study design, data collection, data analysis, data interpretation or writing of the report. The corresponding author had full access to 


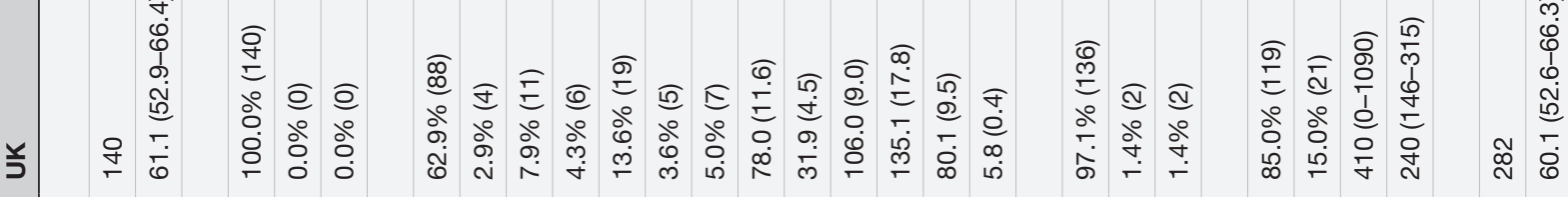

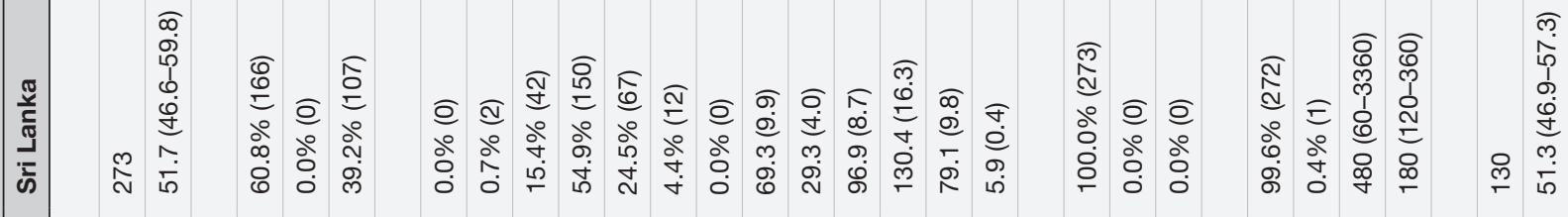

子

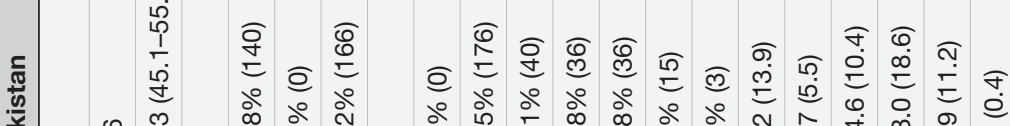

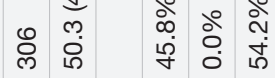

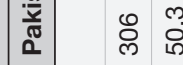

家

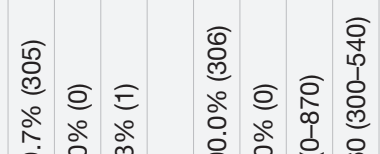

ภ 이

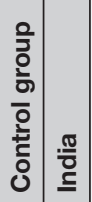

זิ

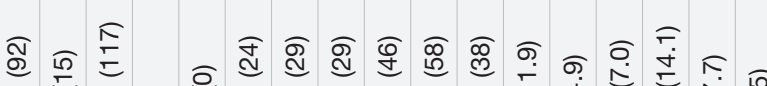

을

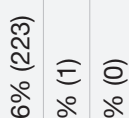

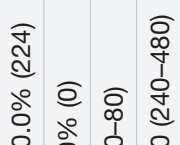

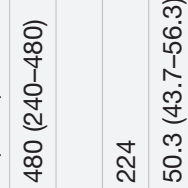

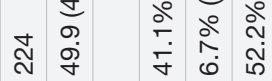

官

๑ே

ํํำ

金)

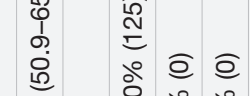

弚

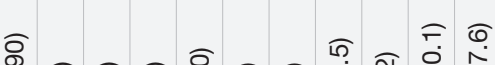

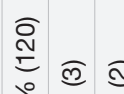

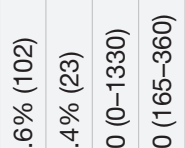

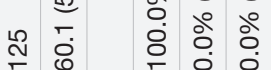

วิ

○े 웅

هั

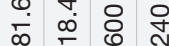

:

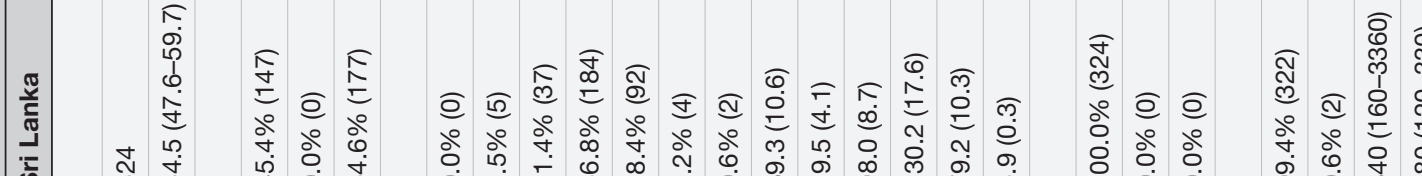

के 窇

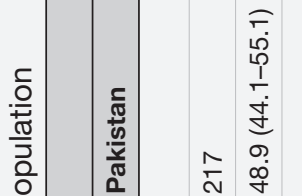

昰

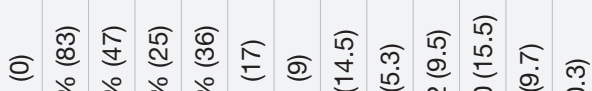

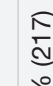

竞

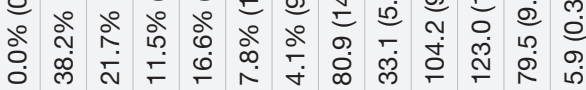
ㅇำ

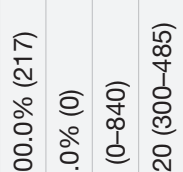
站

๙্ণ

은 व్ర

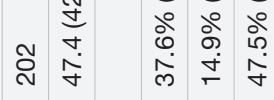

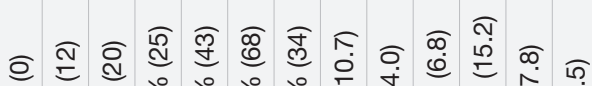

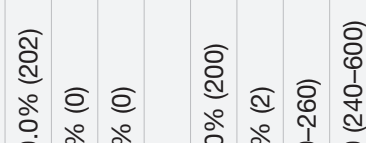

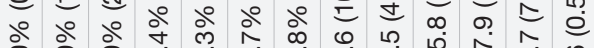

응 ठㅇํㅇ

ஓं 임

$\stackrel{\infty}{\sim} \stackrel{\infty}{\sim}$ 


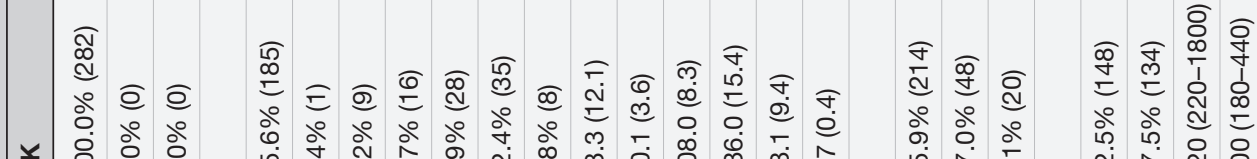

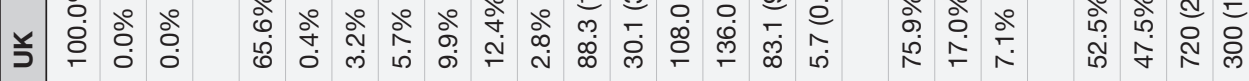

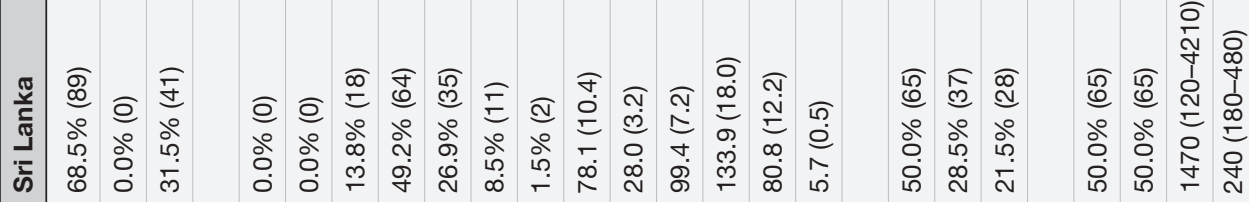

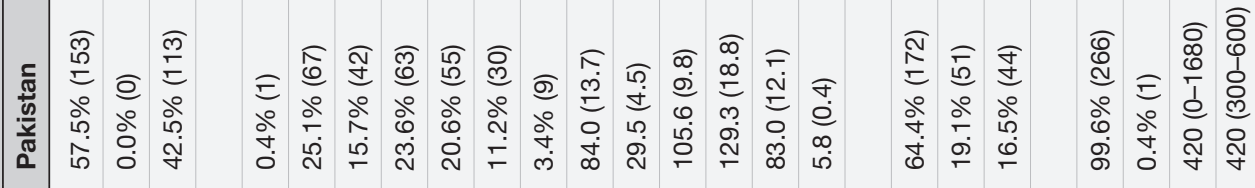

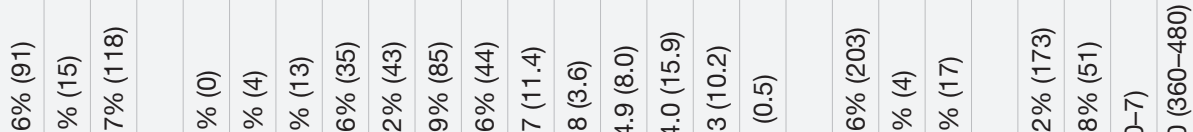

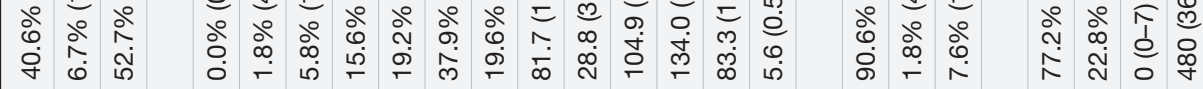

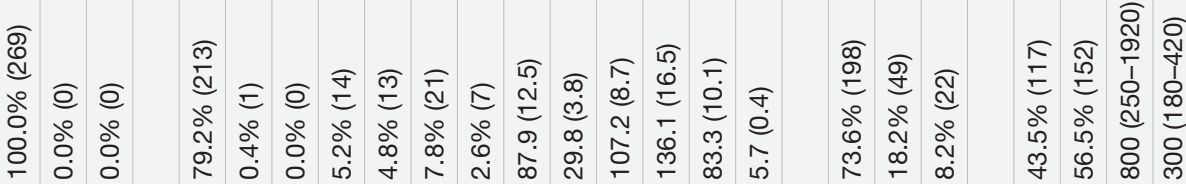
节

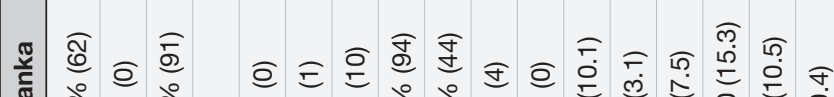

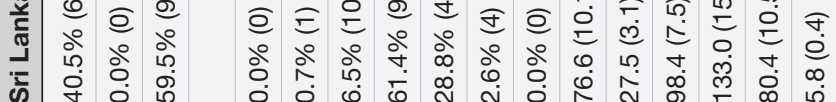

के

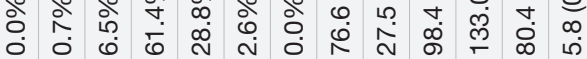

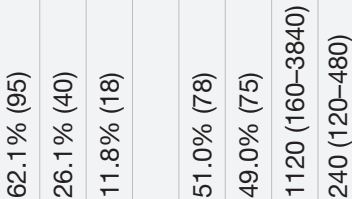

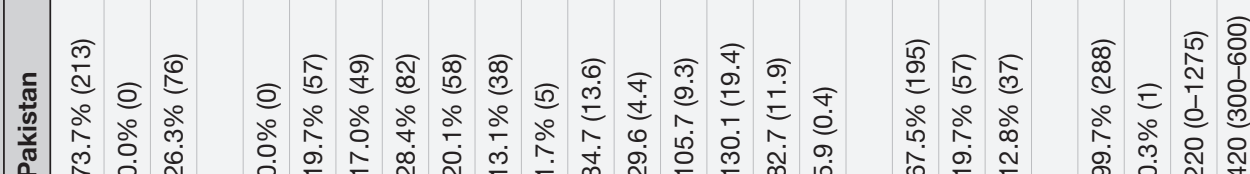
ஸे बं

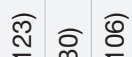

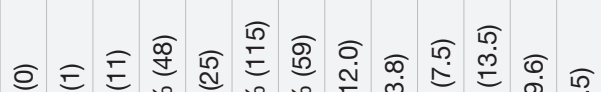

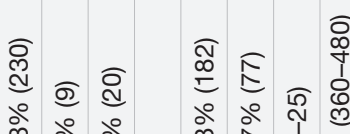

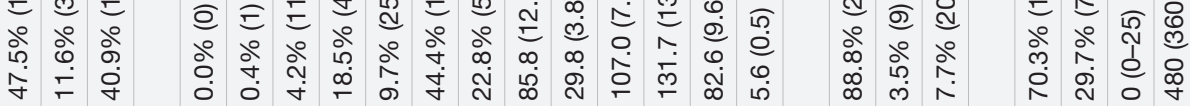
守 $\begin{array}{llll}\infty & 0 & 0 \\ \infty & 0 & 0 \\ \infty & 0 & 0\end{array}$

i
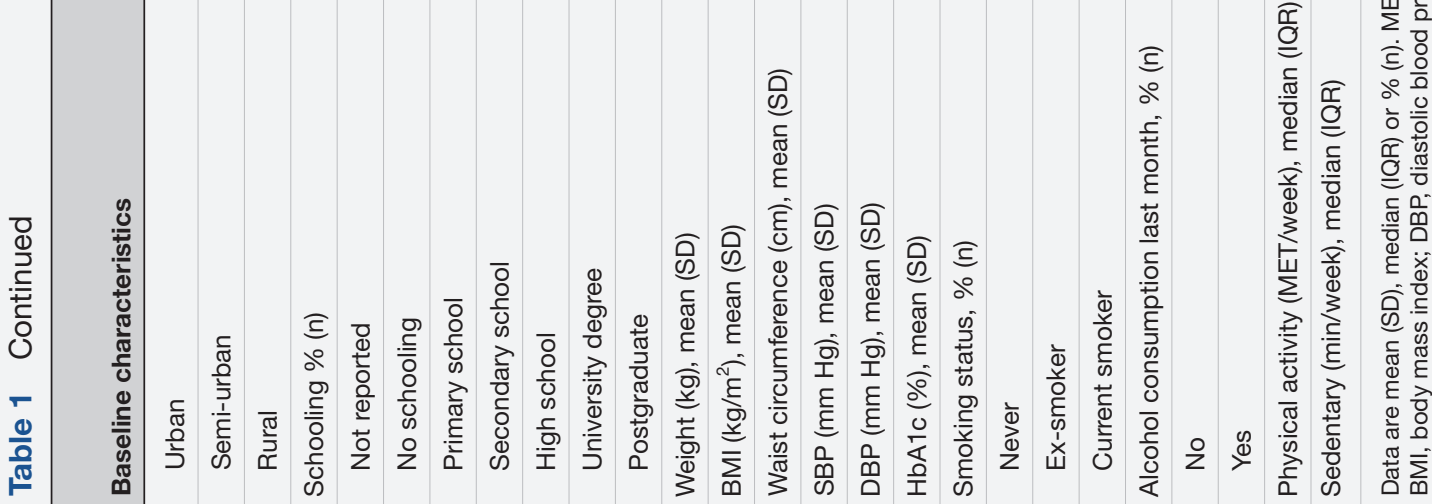


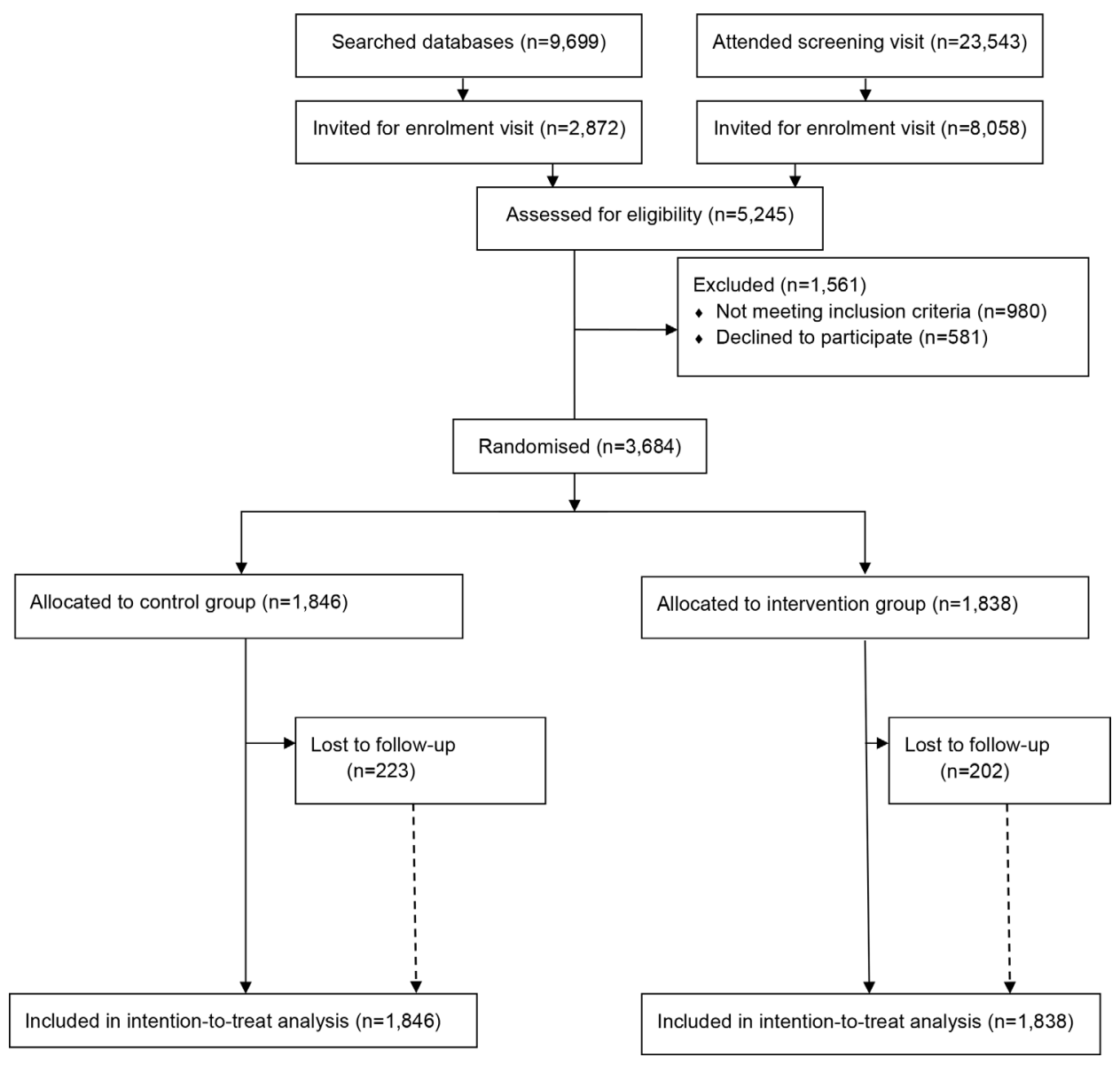

Figure 1 Trial profile of i-Health-T2D study. T2D, type 2 diabetes.

all the data in the study and had final responsibility for the decision to submit for publication.

\section{RESULTS}

Participants were recruited between 15 June 2016 and 05 March 2019. Figure 1 shows the trial profile of the iHealth-T2D study. The opportunistic, community-based recruitment strategies used in the South Asian subcontinent did not enable calculation of response rates. In the UK, the response rate to postal invitation was 22\% (3 132 people attending in response to 14564 letters). Overall, there were 23543 people who attended an iHealth-T2D screening visit. In addition, a database with 9699 South Asians aged between 40 and 70 years comprising the results of recent cardiovascular health assessments was used to screen participants in the UK. Of 33242 people who were screened, 10930 were invited for an assessment of eligibility, where 4263 people met the inclusion criteria and 3684 entered the trial as participants. The participants were randomised to clusters by 120 sites. A total of 1838 participants were assigned to the intervention and 1846 to the control arm, of whom 1636 participants $(89 \%)$ in the intervention and $1623(88 \%)$ in the control arm attended the 1-year follow-up examination.

\section{Baseline characteristics of the studied population}

Table 1 shows the baseline characteristics of women and men by trial arm and country. At baseline, the intervention and control group were comparable in terms of metabolic and lifestyle-related characteristics (online supplemental table 1. Characteristics, however, varied by country and sex; $49.2 \%$ of the participants were woman and the mean enrolment age of participants was 52.8 (SD 8.2) years. Many UK participants did not fill out their education and many Pakistani participants only attended primary school. BMI ranged from $27.5 \mathrm{~kg} / \mathrm{m}^{2}$ among Sri Lankan men to $32.75 \mathrm{~kg} / \mathrm{m} 2$ among Pakistani women. The majority of the participants were non-smokers. The majority of Pakistani men and women did not consume any alcohol, while a quarter of the men in India and half of the men in Sri Lanka and the UK reported alcohol consumption. Median moderate to vigorous-intensity physical activity ranged from zero MET/week among Indian participants to $1470 \mathrm{MET} /$ week among Sri Lankan men.

\section{Study outcomes after 1 year of follow-up}

The weight $(7 \%)$ target was reached by $13.6 \%$ of the participants in the intervention and $6.5 \%$ in the control group, while the waist circumference target $(\geq 5 \mathrm{~cm})$ was met by 30.8 versus $19.4 \%$. Table 2 shows that participants 
Table 2 Weight, waist circumference, blood pressure and $\mathrm{HbA1c}$ between baseline and 12 months

\begin{tabular}{|c|c|c|c|c|c|}
\hline & & Baseline & Follow-up & Adjusted difference $(95 \% \mathrm{Cl})$ & $P$ value \\
\hline \multirow[t]{2}{*}{ Weight (kg) } & Intervention & $80.3(13.7)$ & $78.5(13.8)$ & $-1.1(-1.7$ to -0.6$)$ & $<0.001$ \\
\hline & Control & $79.2(13.5)$ & $78.8(14.0)$ & & \\
\hline \multirow[t]{2}{*}{ Waist circumference $(\mathrm{cm})$} & Intervention & $103.9(9.3)$ & $101.0(10.0)$ & $-1.9(-2.5$ to -1.3$)$ & $<0.001$ \\
\hline & Control & $103.9(9.4)$ & $103.0(9.9)$ & & \\
\hline \multirow[t]{2}{*}{$\mathrm{SBP}(\mathrm{mm} \mathrm{Hg})$} & Intervention & $130.7(16.9)$ & $130.5(17.0)$ & $-0.8(-2.2$ to 0.6$)$ & 0.248 \\
\hline & Control & $131.8(17.1)$ & $132.2(17.5)$ & & \\
\hline \multirow[t]{2}{*}{$\mathrm{DBP}(\mathrm{mm} \mathrm{Hg})$} & Intervention & $81.0(10.2)$ & $80.5(10.2)$ & $-0.7(-1.5$ to 0.2$)$ & 0.130 \\
\hline & Control & $81.6(10.4)$ & $82.0(10.4)$ & & \\
\hline \multirow[t]{2}{*}{$\mathrm{HbA1c}(\%)$} & Intervention & $5.8(0.4)$ & $5.8(0.6)$ & $0.0(-0.1$ to 0.0$)$ & 0.705 \\
\hline & Control & $5.7(0.4)$ & $5.8(0.5)$ & & \\
\hline
\end{tabular}

$\mathrm{N}=1838$ for intervention group; $\mathrm{N}=1846$ for control group. Data are presented as mean and SD. Adjusted group differences are adjusted for stratification variables at randomisation (country and site) confounders (sex and age) and for corresponding baseline value. DBP, diastolic blood pressure; HbA1c, haemoglobin A1c; SBP, systolic blood pressure.

in the intervention group lost more weight and waist circumference than in the control group after 1 year of follow-up. The adjusted mean differences for weight and waist circumference in the intervention group compared with the control group were $-1.1 \mathrm{~kg}(95 \% \mathrm{CI}-1.7$ to -0.6 ; $\mathrm{p}$ value $<0.001)$ and $-1.9 \mathrm{~cm}(95 \% \mathrm{CI}-2.5$ to -1.3 ; $\mathrm{p}$ value $<0.001)$, respectively. In contrast, no statistically significant differences between the two treatment arms were observed for systolic or DBP or for HbAlc.

We observed little evidence for multiplicative interactions between treatment arm and sex, age, setting (the four different countries), waist circumference or HbAlc levels with any of the outcomes (weight; waist circumference; HbA1c; blood pressure; data not shown). However, for participants who lived in Pakistan and/or did not enjoy schooling (groups showed overlap and cannot be disentangled), we found a multiplicative interaction between treatment arm and both systolic and DBP ( $p$ value $=0.04)$. Specifically, among participants who lived in Pakistan and/or did not enjoy schooling, blood pressure increased in both the intervention and the control group, but the increase was lower in the intervention than the control group. (SBP among participants living in Pakistan: intervention arm increased from 127.1 (SD 18.2) to 129.9 (SD 19.7) $\mathrm{mm} \mathrm{Hg}$, while control arm increased from 128.6 (SD 18.6) to 133.8 (SD 20.9) $\mathrm{mm} \mathrm{Hg}$.

Subgroup analyses for participants stratified by inclusion based on raised $\mathrm{HbAlc}$ and/or waist circumference are shown in table 3. Outcomes were similar in both inclusion groups for weight and waist circumference. A numerically larger effect of the intervention on blood pressure was observed for the participants included in the study based on elevated HbAlc levels compared with participants included based on waist circumference. Moreover, a statistically significant decrease in DBP was observed for the HbA1c inclusion group in contrast to the effect observed in the overall group (adjusted group difference HbAlc group $-1.6 \mathrm{~mm} \mathrm{Hg}$ (95\% CI -2.8 to
$-0.4 ; \mathrm{p}$ value $=0.009))$. However, the formal test for effect modification by HbAlc levels was not statistically significant ( $\mathrm{p}$ value $=0.06$ ) .

LSM sessions were mostly completed as planned, the median number of sessions for the intervention group was 18 (IQR 6-22). 665 participants (36\%) completed all 22 , while $1200(65 \%)$ completed at least half of the intervention sessions. For participants who attended every session a $3.37 \mathrm{~cm}$ decrease in waist circumference and $1.86 \mathrm{~kg}$ reduction in weight can be observed (figure 2). This decrease in weight and waist circumference was similar for participants who attended less sessions. Blood pressure and $\mathrm{HbA1c}$, however, depended on the number of sessions attended. A statistically significant difference was observed for participants attending 1-5 sessions (SBP, DBP and HbA1c), participants attending 5-10 sessions (DBP only) and participants attending 11-15 sessions (HbA1c only) compared with those attending all 22 sessions. For instance, HbA1c levels increased by $0.05 \%$ among participants attending all 22 sessions, while it increased by $0.16 \%$ among participants attending $1-5$ sessions ( $p$ value $=0.002$ ).

No adverse events from the intervention were found.

Sensitivity analyses did not identify extreme centres to influence the findings. Complete case analyses showed similar results as the multiple imputation approach.

\section{DISCUSSION}

Our study suggests that a family-based LSM consisting of 22 sessions and delivered by community health workers is effective to decrease weight and waist circumference among South Asians at high risk for T2D, in comparison with usual care. This effect was observed both for participants identified at high risk for T2D based on raised HbAlc and waist circumference, and in South Asians living in low-middle-income country and high-income country settings. No statistically significant effect of the 
Table 3 Outcomes between baseline and 12 months by subgroups of participants enrolled into the trial if they had central obesity and/or elevated HbA1c levels

\begin{tabular}{|c|c|c|c|c|c|}
\hline & & Baseline & Follow-up & Adjusted difference $(95 \% \mathrm{Cl})$ & $P$ value \\
\hline \multicolumn{6}{|c|}{ Inclusion based on central obesity } \\
\hline \multirow[t]{2}{*}{ Weight (kg) } & Intervention & $83.1(12.8)$ & $81.4(13.0)$ & $-1.1(-1.7$ to -0.5$)$ & $<0.0014$ \\
\hline & Control & $81.8(12.9)$ & $81.4(13.6)$ & & \\
\hline \multirow[t]{2}{*}{ Waist circumference $(\mathrm{cm})$} & Intervention & $106.6(7.5)$ & $103.1(9.1)$ & $-1.8(-2.4$ to -1.3$)$ & $<0.001$ \\
\hline & Control & $106.6(7.7)$ & $105.1(9.1)$ & & \\
\hline \multirow[t]{2}{*}{$\mathrm{SBP}(\mathrm{mm} \mathrm{Hg})$} & Intervention & $131.0(16.6)$ & $130.6(16.8)$ & $-0.9(-2.3$ to 0.6$)$ & 0.23 \\
\hline & Control & $132.4(17.0)$ & $132.5(17.1)$ & & \\
\hline \multirow[t]{2}{*}{$\mathrm{DBP}(\mathrm{mm} \mathrm{Hg})$} & Intervention & $81.3(10.1)$ & $80.9(10.1)$ & $-0.6(-1.5$ to 0.3$)$ & 0.19 \\
\hline & Control & $82.2(10.4)$ & $82.4(10.2)$ & & \\
\hline \multirow[t]{2}{*}{$\mathrm{HbA1c}(\%)$} & Intervention & $5.8(0.4)$ & $5.8(0.6)$ & $-0.02(-0.07$ to 0.02$)$ & 0.35 \\
\hline & Control & $5.7(0.4)$ & $5.8(0.5)$ & & \\
\hline \multicolumn{6}{|l|}{ Inclusion based on $\mathrm{HbA} 1 \mathrm{c}$} \\
\hline \multirow[t]{2}{*}{ Weight (kg) } & Intervention & $76.2(14.5)$ & $74.5(14.7)$ & $-1.5(-2.3$ to -0.6$)$ & $<0.001$ \\
\hline & Control & $75.4(13.9)$ & $75.2(14.5)$ & & \\
\hline \multirow[t]{2}{*}{ Waist circumference (cm) } & Intervention & $100.4(10.6)$ & $98.3(11.1)$ & $-2.1(-3.0$ to -1.2$)$ & $<0.001$ \\
\hline & Control & $100.6(11.1)$ & $100.5(11.0)$ & & \\
\hline \multirow[t]{2}{*}{$\mathrm{SBP}(\mathrm{mm} \mathrm{Hg})$} & Intervention & $130.3(17.5)$ & $132.4(18.2)$ & $-1.5(-3.6$ to 0.5$)$ & 0.14 \\
\hline & Control & $131.4(17.2)$ & $130.1(17.2)$ & & \\
\hline \multirow[t]{2}{*}{$\mathrm{DBP}(\mathrm{mm} \mathrm{Hg})$} & Intervention & $80.5(10.4)$ & $79.7(10.5)$ & $-1.6(-2.8$ to -0.4$)$ & 0.009 \\
\hline & Control & $80.6(10.4)$ & $81.6(10.9)$ & & \\
\hline \multirow[t]{2}{*}{ HbA1c (\%) } & Intervention & $6.2(0.1)$ & $6.2(0.7)$ & $-0.01(-0.08$ to 0.06$)$ & 0.69 \\
\hline & Control & $6.2(0.1)$ & $6.2(0.5)$ & & \\
\hline
\end{tabular}

Data are presented as mean and SD. Adjusted group differences are adjusted for stratification variables at randomisation (country and site), confounders (sex and age) and for corresponding baseline value.

DBP, diastolic blood pressure; HbA1c, haemoglobin A1c; SBP, systolic blood pressure.

intervention was observed on blood pressure or HbAlc. However, DBP did decrease among participants identified at high risk for T2D based on the HbA1c inclusion criterion. In addition, the reduction in waist circumference, blood pressure and HbAlc in the intervention arm was dependent on the number of LSM sessions attended.

Weight and waist circumference are important risk factors for T2D. ${ }^{23}{ }^{24}$ Weight loss, especially in the abdominal area, is, therefore, considered an important measure to counteract the development of T2D. ${ }^{25}$ Our study showed that weight and waist circumference decreased in the intervention compared with the control group. Importantly, the effect was dose dependent for waist circumference. Effect sizes were limited, but comparable or somewhat larger than that of other studies. Our study found reductions of $1.1 \mathrm{~kg}$ in weight and $1.9 \mathrm{~cm}$ in waist circumference, while a recent meta-analysis including a total of 1816 South Asian participants reported a reduction of $0.75 \mathrm{~kg}$ and $1.16 \mathrm{~cm},{ }^{6}$ respectively. According to this meta-analysis, modest adiposity changes eventually resulted in a $35 \%$ relative reduction in diabetes incidence. $^{6}$
Our study adds to the already existing evidence that LSM can also be effective among South Asians, if approaches taken for identification of high-risk individuals and delivery of the intervention are low-resource intensive. This makes the intervention especially sustainable and scalable for low-middle-income settings. Most other trials typically used resource-intensive strategies such as oral glucose tolerance tests for risk identification. Our study suggests that the approach of risk identification based on waist circumference and HbAlc are strategies that may work effectively. HbA1c has the advantage over other strategies such as oral glucose-tolerance tests of being a non-fasting assay, which is simpler and cheaper to administer. ${ }^{26}$ Waist circumference is an anthropometric and non-invasive measure, which is a simple, readily available clinical measure of adiposity and can be measured by subjects in the community once adviced. ${ }^{27}$ This makes waist circumference and $\mathrm{HbAlc}$ tools well suited for community-wide risk stratification and opportunistic screening. Delivery of health promotion for prevention of chronic disease has traditionally been the responsibility of physicians and allied health professionals. However, 

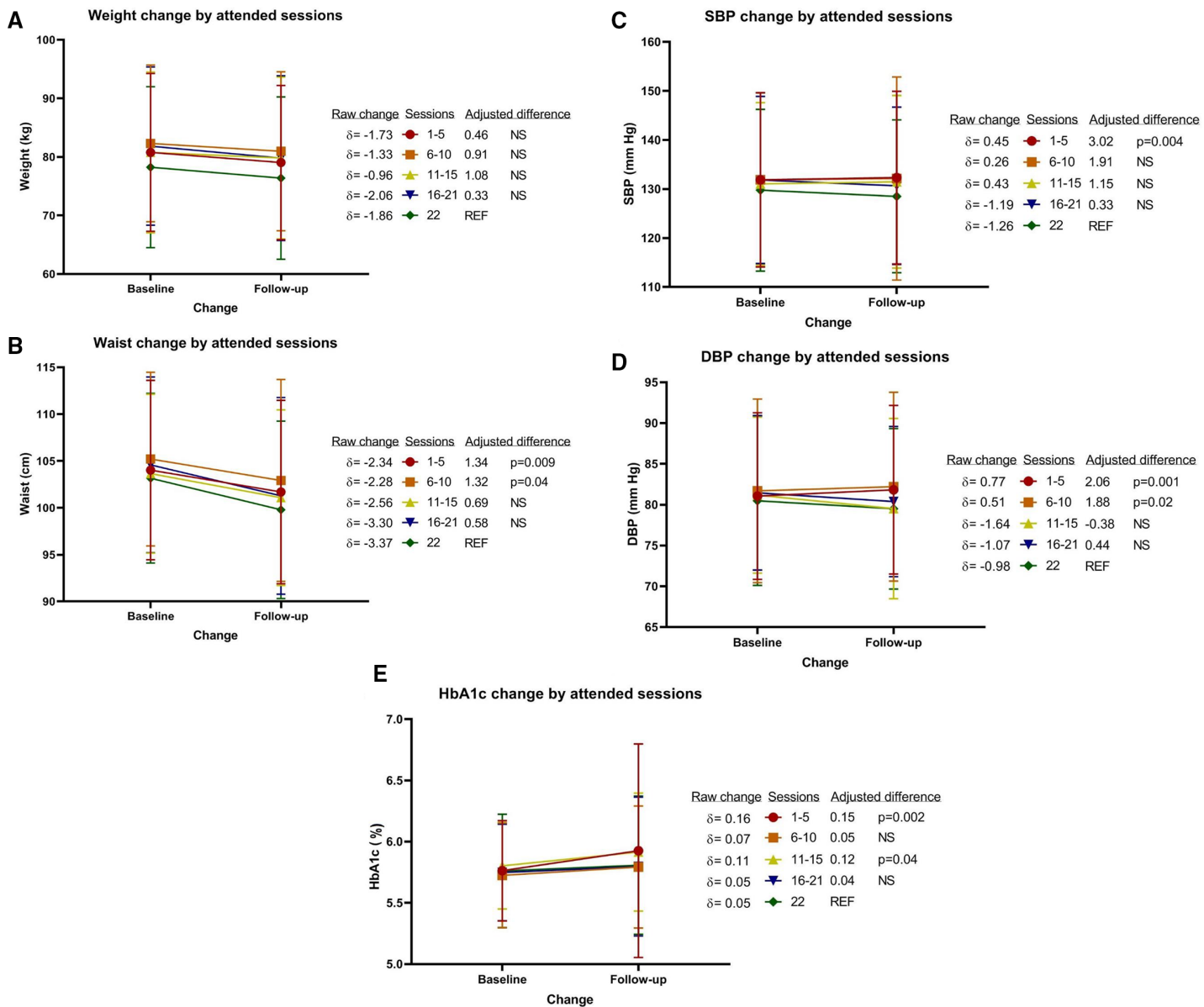

Figure 2 Change in (A) weight, (B) waist circumference, (C) systolic blood pressure, (D) diastolic blood pressure and (E) $\mathrm{HbA1c}$ after 1 year of follow-up. $\mathrm{N}=665$ for the reference group who attended all 22 sessions; $\mathrm{N}=438$ for $1-5$ sessions; $\mathrm{N}=200$ for $6-10$ sessions; $\mathrm{N}=144$ for $11-15$ sessions; $\mathrm{N}=391$ for $16-21$ sessions. Adjusted group differences are adjusted for stratification variables at randomisation (country and site), confounders (sex and age) and for corresponding baseline value. DBP, diastolic blood pressure; HbA1c, haemoglobin A1c; NS, not statistically significant; SBP, systolic blood pressure.

recent research supports the view that community health workers can make an effective contribution in prevention of T2D,,$^{28}$ and these can deliver LSM for prevention of T2D at lower cost, which, thus, provides a possible model for sustainable health promotion in low-middle-income settings. In our study, community health workers with a biological science background and recruited among the local community in which they worked, to ensure natural cultural awareness, were trained.

We did not observe an effect of the intervention on blood pressure or HbAlc in the intervention arm compared with the control arm. However, an effect was observed among participants included in the study based on their HbA1c levels, possibly since HbA1c is likely a better predictor of future T2D diagnosis than raised waist circumference. In addition, dose-response effects by number of attended LSM sessions were observed in the intervention arm. This may imply that the number of sessions is important to the effect of the study, or that participants attending more sessions were more motivated to change their behaviour.

Alcohol consumption and smoking were not very prevalent in this population, and, therefore, we were not able to assess changes in these behaviours. The iHealth-T2D intervention showed an improvement in moderate and vigorous activity levels in both the intervention and control groups but was not able to increase physical activity in the intervention compared with the control group. Moderate to vigorous levels of physical activity were low, especially among participants from India, and may, thus, be a target among South Asians in order to prevent T2D. Future studies could also consider including strength exercise to improve T2D risk. Reduced muscular strength is a modifiable risk factor for T2D and is recently added to guidelines to prevent T2D. ${ }^{29}$ Since muscle strength is relatively low among South Asian populations, this may be a target among these populations as well. ${ }^{30}$

Participants were recruited from 120 sites divided equally between India, Pakistan, Sri Lanka and the UK. The study, thus, included both non-migrant and migrant South Asians. On the South Asian subcontinent, recruitment sites were based in a range of socioeconomic and geographic settings, to increase the generalisability of the findings (though participants in India were mainly reflective of the upper socioeconomic strata, reflecting the recruitment through private hospitals). In addition, 
equal numbers of men and women were included. Nonetheless, if a lifestyle intervention is developed for other regions, elements may need to be adapted to the specific South Asian population targeted, since heterogeneity may exist in both genetic aetiology and in lifestyle factors.

Our study has certain limitations. First, we were not able to evaluate a change in dietary behaviours as they were only available for the intervention arm at baseline. This has limited us in the evaluation of change in explanatory variables underlying the effect of the intervention. Second, it was not possible to blind participants and community health workers to their treatment allocation, as both are actively involved in the intervention. Since the study was only partially blinded, there might be a risk of bias, for instance, during taking measurements. Finally, although our intervention study used low-resource strategies to identify potential participants for the study, LSM is generally resource intensive. However, we sought to reduce costs through group, rather than individual intervention, delivered by trained community health workers, rather than specialist professionals. We did not assess here whether the iHealth-T2D trial was cost-effective, however, this assessment will follow in future studies.

In conclusion, the iHealth-T2D trial is effective to reduce weight, BMI and waist circumference between baseline and 12 months. Since these are important modifiable risk factors for T2D, the iHealth-T2D trial is likely to prevent or delay the onset of T2D in both indigenous and migrant South Asians at high risk of T2D. Finally, the iHealth-T2D trial shows that a family-based intensive LSM programme with a low availability of resources is likely to be effective in preventing T2D among high-risk South Asians in a wide range of settings.

\section{Author affiliations}

${ }^{1}$ Department of Public and Occupational Health, Amsterdam Public Health research institute, Amsterdam UMC Locatie AMC, Amsterdam, The Netherlands

${ }^{2}$ Lee Kon Chian School of Medicine, Nanyang Technological University, Singapore

${ }^{3}$ Department of Epidemiology and Biostatistics, Imperial College London, London, UK

${ }^{4}$ Institute of Endocrinology Diabetes and Metabolism, Max Healthcare, New Delhi, Delhi, India

${ }^{5}$ Department of Endocrinology \& Metabolism, Services Institute of Medical Sciences, Lahore, Punjab, Pakistan

${ }^{6}$ Center for Life Course Health Research, Faculty of Medicine, University of Oulu, Oulu, Finland

${ }^{7}$ Punjab Institute of Cardiology, Lahore, Punjab, Pakistan

${ }^{8}$ Department of Public Health, Faculty of Medicine, University of Kelaniya, Kelaniya, Sri Lanka

${ }^{9}$ Department of Physiology, Faculty of Medicine, University of Colombo, Colombo, Western, Sri Lanka

${ }^{10}$ Department of Endocrinology and Metabolism, Services Institute of Medical

Sciences, Lahore, Punjab, Pakistan

${ }^{11}$ School of Biosciences and Medicine, Faculty of Health and Medical Sciences, University of Surrey, Guildford, UK

${ }^{12}$ Diabetes Research Unit, Faculty of Medicine, University of Colombo, Colombo, Western, Sri Lanka

${ }^{13}$ School of Public Health, Imperial College London, London, UK

${ }^{14}$ Department of Life Sciences, College of Health and Life Sciences, Brunel University, London, UK

${ }^{15}$ Unit of Primary Care, Oulu University Hospital, Oulu, Finland

${ }^{16}$ Institute for Health Policy, Colombo, Sri Lanka
${ }^{17}$ Surrey Health Economics Centre, Department of Clinical and Experimental Medicine, University of Surrey, Guildford, UK

${ }^{18}$ Department of Diabetes and Endocrinology, Imperial College London, London, UK

${ }^{19}$ Department of Metabolism, Digestion and Reproduction, Imperial College London, London, UK

${ }^{20}$ Department of Clinical Medicine, University of Colombo, Colombo, Sri Lanka

${ }^{21}$ London North West University Healthcare NHS Trust, Harrow, UK

${ }^{22}$ National Heart \& Lung Institute, Faculty of Medicine, Imperial College London,

London, UK

Twitter Ravindra Prasan Rannan-Eliya @ravirannaneliya

Acknowledgements We are most grateful to the participants of the iHealth-T2D study, and all the staff members who have taken part in gathering the data of this study.

Contributors GSF, HMG, M-RJ, RPR-E, SA, SJ, AK, PK, KIK, JSK, RW and JCC designed the iHealth-T2D trial. MM and IGMV designed the statistical approach. SJ was the lead investigator in India. SA and KIK were the lead investigators in Pakistan. PK, RW and RPR-E were the lead investigators in Sri Lanka. JCC, JSK and HMG were the lead investigators in the UK. MM performed the data analysis and drafted the first version of the manuscript. SP and IGMV reviewed and edited the manuscript. All authors reviewed the manuscript. All authors read and approved the final manuscript. JCC is the guarantor.

Funding The iHealth-T2D trial was funded by the European Commission (grant award 643774).

\section{Competing interests None declared.}

Patient consent for publication Not applicable.

Ethics approval India: Max Healthcare Institutional Ethic Committee (ref: CT/MSSH/ SKT-2/ENDO/IEC/14-40, date 22 April 2016). Indian Council for Medical Research (ICMR, ref: 55/7/Indo-Foreign-Diab/2014-NCD-II, date 8 February 2016) Pakistan. Punjab Institute of Cardiology Ethical Committee (ref: rtpgme-research-047, date 9 April 2016). Services Institute of Medical Sciences Institutional Review Board (ref: IRB/2016/222/SIMS, 12 March 16) Sri Lanka. University of Colombo Ethics Review Committee (ref: EC-16-063, date 23 May 2016). University of Kelaniya Ethics Review Committee (ref: P/62/05/2016, date 11 May 2016) UK. West Midlands-Solihull Research Ethics Committee (ref: 16/WM/0171, date 14 April 2016).

Provenance and peer review Not commissioned; externally peer reviewed.

Data availability statement Data are available upon reasonable request. Data will be available to others after de-identification, on completion of the research, by application to the Steering Committee.

Supplemental material This content has been supplied by the author(s). It has not been vetted by BMJ Publishing Group Limited (BMJ) and may not have been peer-reviewed. Any opinions or recommendations discussed are solely those of the author(s) and are not endorsed by BMJ. BMJ disclaims all liability and responsibility arising from any reliance placed on the content. Where the content includes any translated material, BMJ does not warrant the accuracy and reliability of the translations (including but not limited to local regulations, clinical guidelines, terminology, drug names and drug dosages), and is not responsible for any error and/or omissions arising from translation and adaptation or otherwise.

Open access This is an open access article distributed in accordance with the Creative Commons Attribution Non Commercial (CC BY-NC 4.0) license, which permits others to distribute, remix, adapt, build upon this work non-commercially, and license their derivative works on different terms, provided the original work is properly cited, appropriate credit is given, any changes made indicated, and the use is non-commercial. See: http://creativecommons.org/licenses/by-nc/4.0/.

\section{ORCID iDs}

Mirthe Muilwijk http://orcid.org/0000-0002-3686-5116

Ravindra Prasan Rannan-Eliya http://orcid.org/0000-0002-5013-2816

\section{REFERENCES}

1 Gujral UP, Pradeepa R, Weber MB, et al. Type 2 diabetes in South Asians: similarities and differences with white Caucasian and other populations. Ann N Y Acad Sci 2013;1281:51-63.

2 Meeks KAC, Freitas-Da-Silva D, Adeyemo A, et al. Disparities in type 2 diabetes prevalence among ethnic minority groups resident in Europe: a systematic review and meta-analysis. Intern Emerg Med 2016;11:327-40. 
3 Ramachandran A, Ambady R, Snehalatha C, et al. Primary prevention of Type 2 diabetes in South Asians--challenges and the way forward. Diabet Med 2013;30:26-34.

4 Lindström J, Louheranta A, Mannelin M, et al. The Finnish diabetes prevention study (Dps): lifestyle intervention and 3-year results on diet and physical activity. Diabetes Care 2003;26:3230-6.

5 Diabetes Prevention Program (DPP) Research Group. The diabetes prevention program (DPP): description of lifestyle intervention. Diabetes Care 2002;25:2165-71.

6 Jenum AK, Brekke I, Mdala I, et al. Effects of dietary and physical activity interventions on the risk of type 2 diabetes in South Asians: meta-analysis of individual participant data from randomised controlled trials. Diabetologia 2019;62:1337-48.

7 Ramachandran A, Snehalatha C, Mary S, et al. The Indian diabetes prevention programme shows that lifestyle modification and metformin prevent type 2 diabetes in Asian Indian subjects with impaired glucose tolerance (IDPP-1). Diabetologia 2006;49:289-97.

8 Ramachandran A, Snehalatha C, Mary S, et al. Pioglitazone does not enhance the effectiveness of lifestyle modification in preventing conversion of impaired glucose tolerance to diabetes in Asian Indians: results of the Indian diabetes prevention Programme-2 (IDPP-2). Diabetologia 2009;52:1019-26.

9 Weber MB, Ranjani H, Staimez LR, et al. The stepwise approach to diabetes prevention: results from the D-CLIP randomized controlled trial. Diabetes Care 2016;39:1760-7.

10 Thankappan KR, Sathish T, Tapp RJ, et al. A peer-support lifestyle intervention for preventing type 2 diabetes in India: a clusterrandomized controlled trial of the Kerala diabetes prevention program. PLoS Med 2018;15:e1002575.

11 Muilwijk M, Loh M, Mahmood S. The iHealth-T2D study: statistical analysis plan for a cluster randomised controlled trial with intensive family-based lifestyle modification programme to reduce type 2 diabetes risk amongst South Asians. medRxiv2020:2020.11.12.20229849.

12 Sampson M, Clark A, Bachmann M, et al. Lifestyle intervention with or without lay volunteers to prevent type 2 diabetes in people with impaired fasting glucose and/or nondiabetic hyperglycemia: a randomized clinical trial. JAMA Intern Med 2021;181:168-78.

13 Kasturiratne A, Khawaja KI, Ahmad S. The iHealth-T2D study: rationale and design of a cluster randomised trial for prevention of type 2 diabetes amongst South Asians with central obesity and prediabetes. medRxiv2020:2020.11.12.20230433

14 McCambridge J, Witton J, Elbourne DR. Systematic review of the Hawthorne effect: new concepts are needed to study research participation effects. J Clin Epidemiol 2014;67:267-77.

15 Onghena P. Resentful Demoralization. In: B.S. E, D.C. H, eds. Encyclopedia of statistics in behavioral science, 2005.
16 Armstrong T, Bull F. Development of the world Health organization global physical activity questionnaire (GPAQ). J Public Health 2006;14:66-70.

17 Gupta SK. Intention-To-Treat concept: a review. Perspect Clin Res 2011;2:109-12.

18 Sullivan TR, White IR, Salter AB, et al. Should multiple imputation be the method of choice for handling missing data in randomized trials? Stat Methods Med Res 2018;27:2610-26.

19 de Boer MR, Waterlander WE, Kuijper LDJ, et al. Testing for baseline differences in randomized controlled trials: an unhealthy research behavior that is hard to eradicate. Int J Behav Nutr Phys Act 2015;12:4

20 Consort, 2010. Available: http://www.consort-statement.org/ consort-2010;

21 Buuren Svan, Groothuis-Oudshoorn K. mice : Multivariate Imputation by Chained Equations in R. J Stat Softw 2011;45:67.

22 Feise RJ. Do multiple outcome measures require $\mathrm{p}$-value adjustment? BMC Med Res Methodol 2002;2:8.

23 Klein S, Allison DB, Heymsfield SB, et al. Waist circumference and cardiometabolic risk: a consensus statement from shaping America's health: association for weight management and obesity prevention; NAASO, the obesity Society; the American Society for nutrition; and the American diabetes association. Diabetes Care 2007;30:1647-52.

24 Ntuk UE, Gill JMR, Mackay DF, et al. Ethnic-Specific obesity cutoffs for diabetes risk: cross-sectional study of 490,288 UK Biobank participants. Diabetes Care 2014;37:2500-7.

25 Haw JS, Galaviz KI, Straus AN, et al. Long-Term sustainability of diabetes prevention approaches: a systematic review and meta-analysis of randomized clinical trials. JAMA Intern Med 2017;177:1808-17.

26 Bonora E, Tuomilehto J. The pros and cons of diagnosing diabetes with A1c. Diabetes Care 2011;34 Suppl 2:S184-90.

27 Obesity in Asia Collaboration, Huxley R, Barzi F, et al. Waist circumference thresholds provide an accurate and widely applicable method for the discrimination of diabetes. Diabetes Care 2007;30:3116-8.

28 Jeet G, Thakur JS, Prinja S, et al. Community health workers for non-communicable diseases prevention and control in developing countries: evidence and implications. PLoS One 2017;12:e0180640.

29 Colberg SR, Sigal RJ, Yardley JE, et al. Physical Activity/Exercise and diabetes: a position statement of the American diabetes association. Diabetes Care 2016;39:2065-79.

30 van der Kooi A-LLF, Snijder MB, Peters RJG, et al. The association of handgrip strength and type 2 diabetes mellitus in six ethnic groups: an analysis of the HELIUS study. PLoS One 2015;10:e0137739. 